\title{
Reducing Thoracic and Lumbar Radiographs in an Urban Emergency Department Through a Clinical Champion Led Quality Improvement Intervention
}

Joshua Sapadin

Albert Einstein College of Medicine

Linelle Campbell

Albert Einstein College of Medicine

Komal Bajaj

Albert Einstein College of Medicine

Joshua Moskovitz ( $\nabla$ joshmoskovitz@gmail.com )

Albert Einstein College of Medicine

\section{Research Article}

Keywords: Low back pain, emergency department, radiographs, x-rays, thoracic, lumbar, appropriate, choosing wisely, medical overuse, quality improvement

Posted Date: October 13th, 2021

DOI: https://doi.org/10.21203/rs.3.rs-959202/v1

License: (c) (i) This work is licensed under a Creative Commons Attribution 4.0 International License. Read Full License 


\section{Abstract}

Background: Low back pain is a common emergency department (ED) complaint that does not always necessitate imaging. Unnecessary imaging drives medical overuse with potential to harm patients. Quality improvement (QI) interventions have shown to be an effective solution. The purpose of this QI intervention was to increase the percentage of appropriately ordered radiographs for low back pain while reducing the absolute number.

Methods: A multi-component intervention led by a clinician champion including staff education, patient education, electronic medical record modification, audit and peer-feedback, and clinical decision support tools was implemented at an urban public hospital Emergency Department. In addition to the total number ordered, Choosing Wisely and American College of Radiology recommendations were used to assess appropriateness of all ED thoracic and lumbar conventional radiographs by chart review over eight months.

Results: The percent of appropriately ordered radiographs increased from $5.8 \%$ to $53.9 \%$ and the monthly number of radiographs ordered decreased from 86 to 47 over the eight-month initiative. There were no compensatory increases in thoracic or lumbar computed tomography (CT) scans during this time frame

Conclusion: A multi-component QI intervention led by a clinician champion is an effective way to reduce the overutilization of thoracic and lumbar radiographs in an urban public hospital emergency department.

\section{Background}

Low back pain is one of the most common presenting complaints in U.S. Emergency Departments (ED). $A_{\text {ttempts }}$ to quantify the prevalence of low back pain suggest there are between two and three million ED visits annually. In addition to a history and physical, evaluation of low back pain in the ED often includes imaging tests. However, overuse of medical imaging for back pain is widely recognized as a problem and a growing concern.

There is potential for patients harm from overutilization through additional testing and imaging, incidental findings, increased length of stay, unnecessary follow-up referrals, invasive procedures, exposure to excess radiation, increased likelihood of surgical interventions, and increased patient and institutional cost. Studies of care for low back pain correlate imaging as an important driver of non-financial costs and have shown this image overutilization fails to improve clinical outcomes. The average costs of thoracic and lumbar radiographs vary widely across the country, ranging from $\$ 100-1000$. Whether paid by the patient, insurance company, or facility itself, inappropriate studies confer little to no benefit to the patient. Unnecessary imaging studies are therefore a conduit for the delivery of low-value care.

The decision to order imaging tests when a patient presents with low back pain depends on a detailed history and physical examination as well as the presence of "red flags", signs and symptoms identifying high risk conditions such as trauma, cancer, infection, or spinal cord involvement. In the absence of "red flag" indicators, non-traumatic acute low back pain less than four weeks in duration generally self resolves and does not necessitate imaging nor intervention. 
Medical overuse is a complex problem, with some clear common drivers. Physician cited motivators are fear of missing a diagnosis, fear of litigation, and perceived improved patient satisfaction. The patient motivators most overwhelmingly cited is their desire for imaging, irrespective of their knowledge of the risks of ionizing-radiation. Evidence suggests multi-component interventions are successful at implementing clinical guidelines that reduce the use of a service. Examples of successes include decreased utilization of antibiotics, radiographs in the ICU, and cardiac imaging.

We hypothesized that a multi-component intervention led by a clinician champion in the ED over the course of eight months would increase the proportion of thoracic and lumbar radiographs ordered that meet appropriateness criteria and decrease the absolute number of those imaging tests ordered.

\section{Methods}

This was a prospective quality improvement (QI) interventional study of all patients receiving conventional lumbar and thoracic radiographic imaging in our Emergency Department from June 2019 to January 2020. The primary outcome was the percentage of appropriately ordered radiographs. The secondary outcome was the absolute number of radiographs completed. The study was approved by the Albert Einstein College of Medicine Institutional Review Board. This manuscript was prepared in accordance with the Standards for Quality Improvement Reporting Excellence (SQUIRE 2.0) guidelines for the reporting of quality improvement work

\section{Study Setting and Population}

\section{Setting}

Safety net urban hospital Emergency Department in the Bronx, New York, with a large Emergency Medicine Residency Program having $>100,000$ annual visits. Staffing in our lower acuity zone (two residents, two advanced practice providers (APPs), and two attendings from $7 \mathrm{am}-11 \mathrm{pm}$ ) where most non-traumatic low back pain patients are evaluated, and where our intervention was more concentrated. The hospital is a level one trauma center serving a limited resource population and a member of the nation's largest public health hospital system. More than $70 \%$ of these patients identify as either Hispanic or African American, and almost a third are uninsured on arrival.

\section{Study Population}

Patients 21 years and older presenting to the ED from June 2019 to January 2020 whom received a thoracic or lumbar conventional radiograph were identified by radiology work list review and chart review. There were no exclusion criteria.

\section{Intervention}

Our intervention had five components: physician education, patient education, a clinical decision support tool, audit and feedback, and Electronic Medical Record (EMR) modification. The entire intervention for this QI initiative was led by one of the authors who served as the clinician champion in the ED. 


\section{Physician and provider education}

Education of the physicians and advance practice providers were done in several stages over time. At resident educational conferences two separate sessions were conducted. "What is medical overuse and why does it create harm?" was presented in May 2019 (one month prior to the start of the intervention) to garner interest in the topic and recruit resident clinician champions, and "Evaluating Low Back Pain in the Urban Emergency Department" in August 2019 (two months into the intervention). The topic was also discussed and reviewed at bi-weekly faculty meetings.

\section{Patient education}

Patient education was implemented in three phases beginning at the start of the intervention (June 2019). First, when registering for care, patient education material in both English and Spanish about low back pain evaluation from the Choosing Wisely website was available for patients to pick up on their own. Second, the same educational material was posted on the walls of patient rooms in the low acuity zone of the ED. Third, face-to-face education of the patients was done by the treating physician. In all phases, patient education consisted of presenting the indications for radiographs, the risks of imaging without red flag indicators as well as expected disease courses.

\section{Clinical decision support tool}

We used the Choosing Wisely (CW) and the American College of Radiology (ACR) recommendations for low back pain imaging to develop "Red Flag" indicators for low back pain imaging in the setting of low back pain in the ED. All providers were given a clinical decision support tool in the form of a best practice advisory (BPA) card (Figure 1) with these Red Flag indicators in their mailboxes and at faculty and at residency conferences. The BPA cards are approximately two by three-inch laminated cards that can easily fit into a lab coat or scrub pocket. In addition, BPA cards were physically attached to the upper right-hand corner of each computer in the ED where providers would be entering orders. The intent of BPA cards was to serve as an easily accessible clinical reminder when determining the need to order imaging for low back pain patients. The BPA cards were redistributed every other month as needed.

\section{Audit and feedback}

At the beginning of each month, the highest utilizers of radiographic imaging for low back pain from the prior month were identified via audit of the EMR: five attending physicians and five residents or Advanced Practice Providers. Feedback was given to each provider in the form of a face-to-face discussion involving a review of the BPA card material. The feedback was conducted by one of the authors based on a prior existing relationship with the highest utilizers.

\section{EMR Modification}

Within the EMR of our ED, we have departmental favorite orders that are available on a quick list intended to save time when placing common orders. We removed the quick-order option for thoracic and lumbar radiographs, thereby necessitating providers to search for the order manually if needed. 


\section{Data Collection and Analysis}

Data on thoracic or lumber radiographs for patients with low back pain was obtained from the Department of Radiology logs of imaging performed in the ED from three months prior to beginning the QI initiative until eight months after. In addition to radiographs, counts of thoracic or lumbar CT scans completed on patients with low back pain seen in the ED were collected during the same time interval. This was done to assess if providers were replacing radiograph orders with CT orders. Magnetic Resonance Imaging is not readily available in our ED, reserved only for emergencies, and typically necessitates an inpatient hospitalization and therefore was not assessed for this project.

Medical record numbers from the logs were used to identify patients in the EMR for evaluation of imaging appropriateness. Using $\mathrm{CW}^{7}$ and $\mathrm{ACR}^{8}$ criteria for imaging in the setting of back pain, we defined appropriateness as back pain lasting less than four weeks with at least one red flag (Figure 1). To determine appropriateness a chart review was done on all patients who received conventional lumbar and thoracic radiographs in our ED using these appropriateness criteria. Chart review was split evenly and conducted by three residents and one attending physician. Each chart reviewer had $10 \%$ of their charts reviewed by a second reviewer to assess inter-rater reliability, of which $100 \%$ was noted.

The proportion of patients with an appropriate thoracic or lumbar radiograph was calculated and reported by month, along with a total count of the number of low back pain patients with a thoracic or lumbar radiographs or CT scan ordered. As a QI initiative, no statistical tests of significance were performed because of the lack of a control group.

\section{Results}

Over the course of the eight-month intervention a total of 432 thoracic and lumbar radiographs were performed and the absolute number of radiographs ordered monthly decreased from 86 to 47 (Figure 2). This compares to an average monthly number of 90 thoracic or lumbar radiographs ordered over the three months prior to the QI initiative. The percent of appropriate radiographs ordered increased from 5.8-53.2\% over the same time period. A separate count of the number of computed tomography (CTs) of the thoracic and lumbar spine performed for low back pain demonstrated no evidence of replacing radiographs with CT scans. (Figure 3)

\section{Discussion}

A multi-component QI intervention led by a clinician champion resulted in a reduction in the number of radiographs ordered to evaluate low-back pain and an increase in the proportion of those ordered that were appropriate. In addition, there was no evidence that these imaging tests were replaced by available alternative imaging. To our knowledge this is the first evaluation of a clinician champion lead initiative that addresses thoracic and lumbar radiograph reduction in the ED setting using a multi-component QI intervention. By engaging clinicians through education, audit and feedback and providing them with a clinical decision support tool, the total number of unnecessary radiographs ordered decreased by almost 
$44 \%$ and of those that were ordered, the proportion that were appropriate increased by 10 -fold. Over eight months we demonstrated through a multi-component intervention that this could be addressed and substantially reduced.

Our results are consistent with previous studies which highlight evidence that QI interventions can address the issue of reducing image utilization. A QI intervention at a high-volume pediatric ED in California used similar methods to reduce abdominal radiographs for patients presenting with constipation. Relying heavily on physician education in the form of lectures, and audit and feedback, they reduced the number of pediatric patients that received unnecessary abdominal radiographs by $50 \%$ over a 12 -month period. Another QI intervention in a pediatric ED sought to reduce chest radiographs for patients presenting with bronchiolitis. They employed physician education lectures and posted imaging guidelines at each computer station, and reduced radiographs for bronchiolitis patients by $44 \%$ at the end of a four-month period.

The dissemination of official guidelines alone may lead to successful outcomes in QI interventions. However, it has been shown that the addition of clinician champions led to higher rates of success. A literature review studying interventions that attempted to reduce low value care defined by Choosing Wisely found that interventions led by clinician champions reached their intended outcomes $71 \%$ of the time. This underscores the importance of clinician-led initiatives when attempting to improve quality of care.

Concerned the reduction of radiographs could have the unintended consequence of an increase in CT scans of the thoracic and lumbar spine we assessed the absolute number ordered as well. As a level one trauma center that does a significant number of these CT scans, there were no demonstrated special cause variation around the median during our QI project. Further, the number of radiographs in the three months prior to the intervention demonstrate stable common cause variation.

\section{Limitations}

A limitation of this intervention is the lack of a control group. Without a control, we cannot be certain that this was not just a natural phenomenon that would have occurred without the intervention. ED volumes are dynamic and vary monthly due to multiple factors, and our study period was limited from June to January in an academic emergency department, making it possible that other factors could affect the number of radiographs ordered. Low rates of radiograph appropriateness prior to the intervention could be due to poor documentation, but there was demonstrated consistent improvement at the same time of absolute reduction. It is also possible that the observed impact of our intervention could be explained by the Hawthorn effect, in which one's awareness they are being audited leads to changes in their behavior to improve their performance.

\section{Conclusion}

We conclude a multi-component intervention led by a clinician champion is an effective means to reduce utilization of thoracic and lumbar conventional radiographic imaging in a public hospital ED for patients 
with non-traumatic acute low back pain. Further research is needed to validate these findings and explore the sustainability of these results.

Additional research quantifying unnecessary imaging to subsequent incidental findings, procedures, followup, and lengths of stay in the hospital would further the value of using QI interventions to improve the delivery of high-quality care.

Healthcare expenditures are continually on the rise, and with an aging population this will continue to increase. Reducing medical overuse in the ED is an opportunity to reduce the burden of disease on patients, facilities, and systems. While ED visits are increasing, ED imaging is increasing disproportionately faster. This leads to opportunities for intervention and improved patient outcomes.

\section{Abbreviations}

ACR

American College of Radiology

APP

Advanced Practice Providers

BPA

Best Practice Advisory

CT

Computed Tomography

CW

Choosing Wisely

ED

Emergency Department

EMR

Electronic Medical Record

QI

Quality Improvement

\section{Declarations}

Ethics approval and consent to participate

Consent was not obtained from study participants as this was a chart review quality improvement initiative. The study was approved by the Albert Einstein College of Medicine Institutional Review Board under the waiver of informed consent and HIPAA authorization \#069626.

Consent for publication

Not applicable 
Availability of data and material

The datasets used and/or analyzed during the current study are available from the corresponding author on reasonable request.

Competing Interests

None

Funding

Robert Wood Johnson Foundation Grant \#75607

Authors' Contributions

JBM, LC, KB, JS conceived the study. JBM, LC supervised the conduct of the trial and data collection, managed the data, including quality control and analysis. JBM, KB, JS analyzed the data. JBM, JS drafted the manuscript, and all authors contributed substantially to its revision. JBM takes responsibility for the paper as a whole.

Acknowledgements

Jeffrey Kimm, MD

Michelle Sachet, MD

Michael X Parchman, MD, MPH

\section{References}

1. National Center for Health Statistics. (2017). National Ambulatory Medical Care Survey: 2017 State and National Summary Tables. Centers for Disease Control and Prevention. https://doi.org/10.1080/0308107031000075690

2. Friedman BW, Chilstrom M, Bijur PE, Gallagher EJ. Diagnostic testing and treatment of low back pain in United States emergency departments: a national perspective. Spine (Phila Pa 1976). 2010 Nov 15;35(24):E1406-11. doi: 10.1097/BRS.0b013e3181d952a5. PMID: 21030902; PMCID: PMC2982879.

3. Mafi, J. N., McCarthy, E. P., Davis, R. B., \& Landon, B. E. (2013). Worsening trends in the management and treatment of back pain. JAMA Internal Medicine. https://doi.org/10.1001/jamainternmed.2013.8992

4. Newton, E. H. (2017). Addressing overuse in emergency medicine: evidence of a role for greater patient engagement. Clinical and Experimental Emergency Medicine. https://doi.org/10.15441/ceem.17.233

5. Chou, R., Fu, R., Carrino, J. A., \& Deyo, R. A. (2009). Imaging strategies for low-back pain: systematic review and meta-analysis. The Lancet. https://doi.org/10.1016/S0140-6736(09)60172-0 
6. https://www.healthcost.com/search/72080/New\%20York,\%20USA/43.2994285,-74.21793260000001 Accessed May $21^{\text {st }}, 2021$

7. Chou, R., Qaseem, A., Snow, V., Casey, D., Cross, T. J., Shekelle, P., \& Owens, D. K. (2007). Diagnosis and treatment of low back pain: A joint clinical practice guideline from the American College of Physicians and the American Pain Society. In Annals of Internal Medicine. https://doi.org/10.7326/0003-4819147-7-200710020-00006

8. Griffith, J., Monkman, H., Borycki, E., \& Kushniruk, A. (2015). Physician Experiences with Perceived Pressure to Order Diagnostic Imaging Services. Studies in Health Technology and Informatics. https://doi.org/10.3233/978-1-61499-574-6-20

9. Haran JP, Goulding M, Campion M, Scully G, Chandra A, Goldberg R, Day A, McLendon E, Clark MA. Reduction of Inappropriate Antibiotic Use and Improved Outcomes by Implementation of an AlgorithmBased Clinical Guideline for Nonpurulent Skin and Soft Tissue Infections. Ann Emerg Med. 2020 Jul;76(1):56-66. doi: 10.1016/j.annemergmed.2019.12.012. Epub 2020 Feb 13. PMID: 32063343.

10. Scott, J., Waite, S., \&amp; Napolitano, A. (2021). Restricting daily chest radiography in the intensive care unit: Implementing evidence-based medicine to decrease utilizationt. Journal of the American College of Radiology, 18(3), 354-360. https://doi.org/10.1016/j.jacr.2020.05.035

11. Bhatia RS, Milford CE, Picard MH, Weiner RB. An educational intervention reduces the rate of inappropriate echocardiograms on an inpatient medical service. JACC Cardiovasc Imaging. 2013; 6:545-555. doi: 10.1016/j.jcmg.2013.01.010.

12. Louise Davies, Daisy Goodman, Paul Batalden, Frank Davidoff, David Stevens; Squire 2.0 (Standards for Quality Improvement Reporting Excellence): Revised Publication Guidelines from a Detailed Consensus Process. Am J Crit Care h2November 2015; 24 (6): 466-473. doi: https://doi.org/10.4037/ajcc2015455

13. Foundation, A. B. I. M. (2018, October 31). Imaging tests for lower-back pain: Choosing wisely. Choosing Wisely | Promoting conversations between providers and patients. Retrieved September 23, 2021, from https://www.choosingwisely.org/patient-resources/imaging-tests-for-lower-back-pain/.

14. https://www.choosingwisely.org/clinician-lists/acep-lumbar-spine-imaging-in-the-ed/ Accessed May 28,2021

15. https://acsearch.acr.org/docs/69483/Narrative/ Accessed May 28, 2021

16. Moriel, Gabriela, et al. "Reducing Abdominal Radiographs to Diagnose Constipation in the Pediatric Emergency Department." The Journal of Pediatrics, vol. 225, 2020, doi:10.1016/j.jpeds.2020.06.028.

17. Reiter, Joel, et al. "A Quality Improvement Intervention to Reduce Emergency Department Radiography for Bronchiolitis." Respiratory Medicine, vol. 137, 2018, pp. 1-5., doi:10.1016/j.rmed.2018.02.014.

18. Cliff BQ, Avanceña ALV, Hirth RA, Lee SD. The Impact of Choosing Wisely Interventions on Low-Value Medical Services: A Systematic Review. Milbank Q. 202h2Aug 17. doi: 10.1111/1468-0009.12531. Epub ahead of print. PMID: 34402553

19. Iglehart, J. K. (2006). The new era of medical imaging - Progress and pitfalls. New England Journal of Medicine. https://doi.org/10.1056/NEJMhpr061219 


\section{No RED Flags, No RADS! In the absence of red flags: trauma, or worsening neuro Sxs imaging is RARELY helpful for back pain}

\begin{tabular}{|l|l|}
\hline \multicolumn{2}{|c|}{ Red Flag History and Physical Examination Findings } \\
\hline Age $<20$ or $>50$ & \multicolumn{1}{c|}{ Concern } \\
\hline H/o Cancer & Cancer \\
\hline H/o unexplained weight loss & Metastasis \\
\hline Persistent fevers or night sweats & Cancer \\
\hline $\begin{array}{l}\text { - Immunocompromised } \\
\text { - Prolonged steroid use } \\
\text { - IV drug use }\end{array}$ & Epidural abscess, Osteomyelitis \\
\hline $\begin{array}{l}\text { Recent bacterial infection or } \\
\text { bacteremia }\end{array}$ & Osteomyelitis, Discitis \\
\hline Known aortic Aneurysm & $\begin{array}{l}\text { Seeding spine or paravertebral } \\
\text { structures }\end{array}$ \\
\hline Motor neurologic deficit & Retroperitoneal rupture \\
\hline $\begin{array}{l}\text { - Urinary Retention } \\
\text { - Bowel incontinence } \\
\text { - Saddle anesthesia }\end{array}$ & Cord or root compression \\
\hline
\end{tabular}

American College of

Emergency Physicians

\section{Figure 1}

Best Practice Advisory card containing red flag indicators for imaging of low 


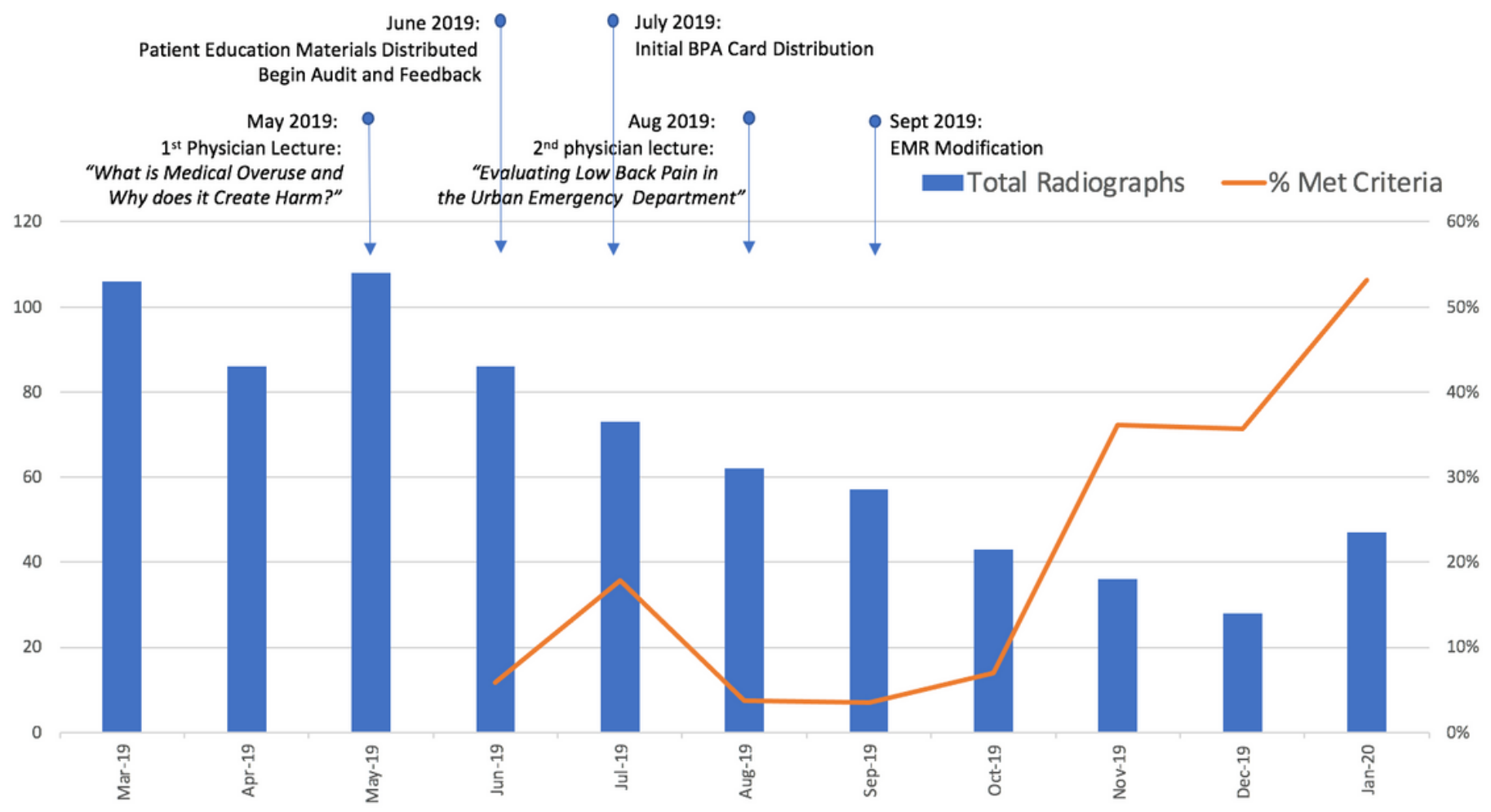

Figure 2

Monthly Radiographs and Percent that Met Appropriateness Criteria 


\section{—Total Radiographs —Total CTs}

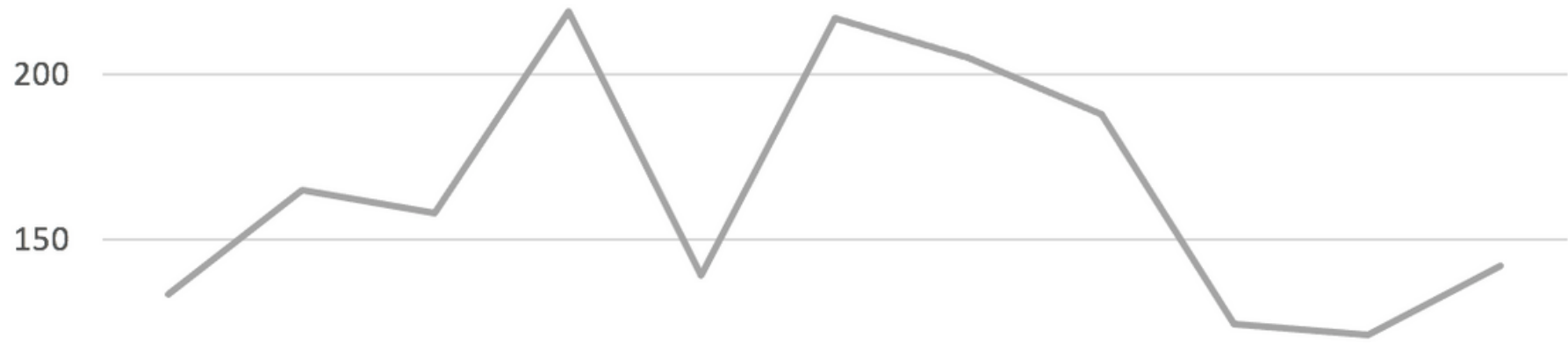

100

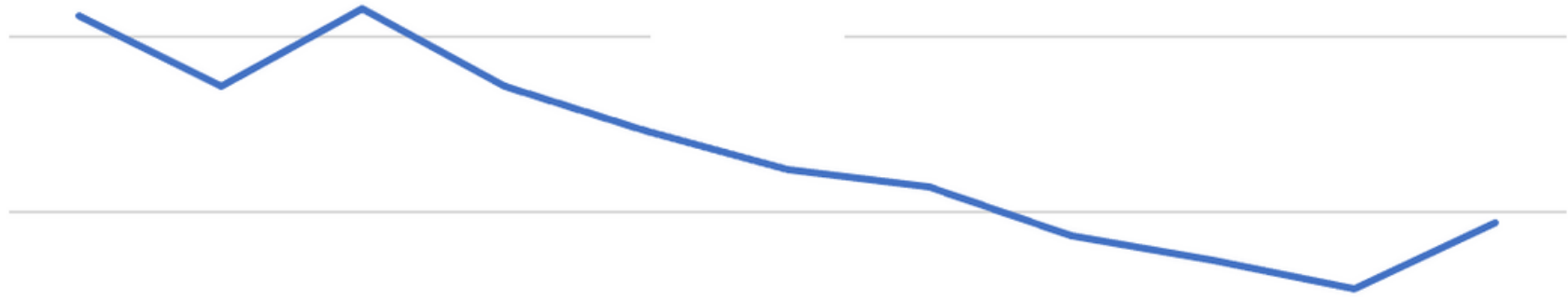

0

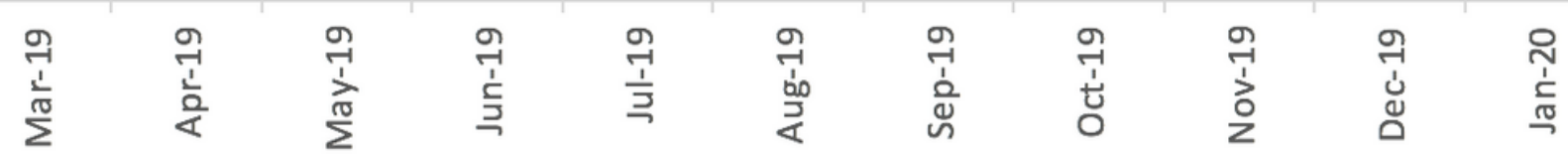

Figure 3

Absolute Monthly Radiographs and CT scans 